\title{
Consensus under Communication Delays
}

\author{
Alexandre Seuret, Dimos V. Dimarogonas and Karl H. Johansson
}

\begin{abstract}
This paper deals with the consensus problem under communication network inducing delays. It is well-known that introducing a delay leads in general to a reduction of the performance or to instability due to the fact that timedelay systems are infinite dimensional. For instance, the set of initial conditions of a time-delay system is not a vector but a function taken in an interval. Therefore, investigating the effect of time-delays in the consensus problem is an important issue. In the present paper, we assume that each agent receives instantaneously its own state information but receives the state information from its neighbors after a constant delay. Two stability criteria are provided based on the frequency approach and on Lyapunov-Krasovskii techniques given in terms of LMI. An analytic expression of the consensus equilibrium which depends on the delay and on the initial conditions taken in an interval is derived. The efficiency of the method is tested for different network communication schemes.
\end{abstract}

\section{INTRODUCTION}

Algorithms for consensus of multi-agent systems is a field that has gained increasing attention in the last few years, due to its applications in multi-robot systems [11], averaging in communication networks [17] and formation control [1]. Several results have appeared in recent literature that consider systems with different motion models, symmetry of communication and network interactions. A recent review of the vast literature in the field can be found in [11]

In this paper we examine a particular case of the consensus problem when the information exchange between the communicating agents has inherit time-delays. In particular, each agent is assumed to have access to the information of its own state with no delays, but can only consider delayed information of the states of its neighbors. The purpose of this paper is to study the stability of such a system with respect to the value of the delay and then to determine the equilibrium point of the consensus problem. The delays of the proposed controller model various phenomena of networked systems such as transmission delays on the transfer of data between each agent and its neighbors, packet losses in wireless communication networks and inaccurate sensor measurements. Moreover, delays can result from sampling. As shown in [2], a sampled signal can be seen as a delayed signal with a particular delay $\tau(t)=t-t_{k}$, which is discontinuous and whose derivative is equal to 1 almost every time. As it is not clear that all the agents have synchronized clocks,

This work was not supported by any organization

Alexandre Seuret, Dimos V. Dimarogonas and Karl H. Johansson are with the ACCESS Linnaeus Center, Royal Institute of Technology, Stockholm, Sweden. seuret, dimos, kallej@kth.se

The work was partially supported by European Commission through the HYCON Network of Excellence, the Swedish Foundation for Strategic Research and by the Swedish Research Council. the assumption that these sampling delays are known is not satisfied in a general case.

A similar model to the one treated in the current paper was used in [7], [8]. The author uses a nonsmooth Lyapunov approach and treats the case of time-varying graphs with time-delays. However, that paper treated the time-delayed model as a simple extension of the non-delayed case, not considering the effect that initial conditions have in the resulting consensus equilibrium. In this paper, we provide an analytic expression of the resulting consensus point and relate it to the initial conditions of the time-delayed model. We should also note that the model used in the paper is different than the one used in [10] that assumes that each agent has the same delay in its own information and the information of its neighbors. Thus, the stability analysis and results of the current paper refer to a different model, and are thus different than the corresponding ones in [10]. In particular, we provide stability conditions using LyapunovKrasovskii techniques which are given in terms of LMI. The communication topology is asymmetric and the symmetric case is treated as a special case of the main theory. Results on stability of discrete-time consensus algorithms with communication delays have already appeared in [16]. The difference in the current paper is that continuous-time models of agent dynamics are considered.

The rest of the paper is organized as follows: Section II includes the necessary background on consensus and timedelay systems and presents the problem treated in this paper. The stability analysis of the closed-loop system is given in Section III which includes both the cases of asymmetric and symmetric communication topologies. Section IV includes illustrating simulation examples while Section V summarizes the results of the paper and indicates current research efforts.

Notation: Throughout the paper, the superscript ' $T$ ' stands for matrix transposition, $R^{n}$ denote the $n$-dimensional Euclidean space, $R^{n \times m}$ is the set of $n \times m$ real matrices. The notation $P>0$ for $P \in R^{n \times n}$ means that $P$ is a symmetric and positive definite matrix. $I$ represents the identity matrix. Finally, for any matrix $M$, the notation $(M)_{i}$ denotes the $i^{t h}$ row of the matrix $M$.

\section{Problem Statement}

\section{A. Consensus Problems}

We first review the original non delayed consensus problem for $N \in \mathbb{N}$ agents with fixed but non necessarily symmetric communication links. The open-loop dynamics are given by:

$$
\dot{x}_{i}(t)=u_{i}(t), \quad i \in\{1, \ldots, N\} .
$$


The consensus control law with no time delays in [10] is given by

$$
u_{i}(t)=\sum_{j \in \mathcal{N}_{i}} a_{i j}\left(x_{j}(t)-x_{i}(t)\right), \quad i \in\{1, \ldots, N\} .
$$

where $\mathcal{N}_{i}$ represents the set of agents which are connected to agent $i$ and is called agent $i$ 's communication set. The gains $a_{i j}$ are positive scalar. Note that the communication is not necessarily symmetric, which means $a_{i j} \neq a_{j i}$.

The closed-loop system is written in stack vector form as

$$
\dot{x}=-L x
$$

where $x=\left[x_{1}, \ldots, x_{N}\right]^{T}$ is the stack vector of all agents' states and $L$ is the Laplacian matrix [3] of the communication graph $G$ of the network, which is defined based on the communication sets $\mathcal{N}_{i}$.

A brief background on the construction of the Laplacian matrix is given in the sequel. For the graph $G$ with $N$ vertices and edge set given by $E=\left\{(i, j): j \in \mathcal{N}_{i}\right\}$ the adjacency matrix $A=A(G)=\left(a_{i j}\right)$ is the $N \times N$ matrix given by $a_{i j}=1$, if $(i, j) \in E$ and $a_{i j}=0$, otherwise. If there is an edge connecting two vertices $i, j$, i.e. $(i, j) \in E$, then $i, j$ are called adjacent. If there is a path between any two vertices of the graph $G$, then $G$ is called strongly connected in the case of directed, and simply connected in the case of undirected graphs. The degree $d_{i}$ of vertex $i$ is defined as the number of its neighboring vertices, i.e. $d_{i}=\# j:(i, j) \in E$. Let $\Delta$ be the $N \times N$ diagonal matrix of $d_{i}$ 's. The Laplacian of $G$ is the matrix $L=\Delta-A$. For an undirected graph the Laplacian matrix is symmetric positive semidefinite. When the directed graph is strongly connected, the Laplacian has a single zero eigenvalue and the corresponding eigenvector is the vector of ones, $\overrightarrow{\mathbf{1}}$. This result was established in [10]. For the case of undirected graphs, a necessary and sufficient condition for zero to be a simple eigenvalue of the Laplacian matrix, is that the undirected graph is connected.

The main result of [10] states that a sufficient condition for the system (3) to reach consensus is that the underlying communication graph $G$ is strongly connected.

\section{B. Time-delay systems}

The tools from time-delay systems used in the sequel are reviewed in the next paragraphs. Consider the linear system with constant delay:

$$
\left\{\begin{array}{l}
\dot{x}(t)=A^{0} x(t)+A^{\tau} x(t-\tau), \\
x(\theta)=\phi(\theta), \quad \forall \theta \in\left[\begin{array}{ll}
-\tau, \quad 0
\end{array}\right]
\end{array}\right.
$$

where $x \in R^{n}$ is the state variable and $A^{0}$ and $A^{\tau}$ are constant matrices with appropriate dimension. The function $\phi$ corresponds to the set of initial conditions considered over the interval $[-\tau, 0]$. Several conditions have been provided the stability of the system (4) [4], [9],[12]. In this paper, we will focuss on the following lemma:

Lemma 1: ([9], Corollary 5.5, pp222) The system (4) is asymptotically stable for all delays $\tau \in[0, \bar{\tau}]$ if:

- $1+\lambda_{k}\left(A^{\tau}\right) \frac{1-e^{s \bar{\tau}}}{s} \neq 0$, for all $s \in C^{+}$and $k=1, . ., N$, and
- there exist symmetric and positive-definite matrices $P, S$ such that the following matrix is negative-definite:

$$
\left[\begin{array}{cc}
M_{11} & \left(A^{0}+A^{\tau}\right)^{T} P A^{\tau} \\
\left(A^{\tau}\right)^{T} P\left(A^{0}+A^{\tau}\right) & -\bar{\tau} S .
\end{array}\right]
$$

where $M_{11}=\left(A^{0}+A^{\tau}\right)^{T} P+P\left(A^{0}+A^{\tau}\right)+\bar{\tau} S$.

Proof: The proof is based on the Lyapunov functional:

$$
\begin{aligned}
V(x)= & \left(x^{T}(t)+\int_{t-\tau}^{t} x^{T}(\theta) d \theta A^{\tau T}\right) P \\
& \left(x(t)+A^{\tau} \int_{t-\tau}^{t} x(\theta) d \theta\right)+\int_{-\tau}^{0} \int_{-\tau}^{0} x(\xi)^{T} S x(\xi) d \xi
\end{aligned}
$$

Remark 1: Lemma 1 is delay-dependent, i.e., the delay $\bar{\tau}$ appears in it and provides sufficient but not necessary condition for asymptotic stability. Based on the conditions of Lemma 1, it is possible to maximize the upper bound $\bar{\tau}$ such that the system is still stable.

\section{Problem statement}

In this paper the following problem in addressed. From the point of view of agent $i$, the value of $x_{i}$ is provided by embedded sensors. Then the state $x_{i}$ is available at every time $t$ without any delay. However the data coming from the other agents $j \in \mathcal{N}_{i}$ are received by agent $i$ after a time-delay caused by the various reasons given in the introduction. Consider further as an approximation that all the communication delays are constant and equal to $\tau$ which can be assimilated as an average delay. We then derive the control law (6):

$$
\dot{x}_{i}(t)=\sum_{j \in \mathcal{N}_{i}} a_{i j}\left(x_{j}(t-\tau)-x_{i}(t)\right) \quad i \in\{1, \ldots, N\},
$$

and moreover assume that there exists a constant and positive scalar $\mu$ such that:

$$
\sum_{j \in \mathcal{N}_{i}} a_{i j}=\mu, \quad i \in\{1, \ldots, N\} .
$$

Define now the vector $x(t)=\left[x_{1}(t), . ., x_{N}(t)\right]^{T}$. Then (6) can be written as:

$$
\dot{x}(t)=-\mu I x(t)+A x(t-\tau),
$$

where $A$ is the adjacency matrix of the communication graph.

Remark 2: An extension to the multiple delays case would consider the following system:

$$
\dot{x}_{i}(t)=\sum_{j \in \mathcal{N}_{i}} a_{i j}\left(x_{j}\left(t-\tau_{i j}\right)-x_{i}(t)\right) \quad i \in\{1, \ldots, N\} .
$$

Remark 3: The reader can notice the similarity of (7) and (4). We can thus use results on stability of the time-delay system (4) to study the stability of the consensus delay system (7).

Remark 4: In contrast to (8), the following delayed version of the consensus algorithm is considered in [10]

$$
\dot{x}_{i}(t)=\sum_{j \in \mathcal{N}_{i}} a_{i j}\left(x_{j}\left(t-\tau_{i j}\right)-x_{i}\left(t-\tau_{i j}\right)\right),
$$

where $i \in\{1, \ldots, N\}$ and $\tau_{i j}>0$ are constant delays. Thus the analysis of that paper is different than that of the paper in hand. The model used in the current paper is more realistic 
for cases such as the one described above, where each agent has access to its own state through embedded sensors, and delays are only present in the interagent information exchange.

By the Leibnitz formula, we have $x(t-\tau)=x(t)-$ $\int_{t-\tau}^{t} \dot{x}(s) d s$, for all differentiable functions $x$. System (7) can be rewritten as:

$$
\dot{x}(t)=(-\mu I+A) x(t)-A \int_{t-\tau}^{t} \dot{x}(s) d s .
$$

Note that the matrix $-\mu I+A$ corresponds to the Laplacian matrix. This representation is a way to understand how the delay affects the consensus problem.

\section{Definition of an appropriate model}

Knowing that the vector $\overrightarrow{\mathbf{1}}$ is an eigenvector associated to the eigenvalue 0 of the Laplacian matrix, it is possible to find a change of coordinates such that $x=W z$ and:

$$
U(-\mu I+A) W=\left[\begin{array}{cc}
B & \overrightarrow{0} \\
\overrightarrow{0}^{T} & 0
\end{array}\right]
$$

where $U=\left[\begin{array}{l}U_{1} \\ U_{2}\end{array}\right]=W^{-1}$ and $U_{2}=(U)_{N}$. In the case of a symmetric matrix $A$, the rest of the Laplacian eigenvalues are all positive. We denote them by $0<\lambda_{2} \leq \ldots \leq \lambda_{N}$. It thus means that $B$ is a diagonal matrix with $-\lambda_{i}$.

The following lemma provides an appropriate way to rewrite (9) based on the properties of the matrix $L$.

Lemma 2: The system (9) can be rewritten in the following way:

$$
\begin{array}{r}
\dot{z}_{1}(t)=-\mu I z_{1}(t)+(B+\mu I) z_{1}(t-\tau), \\
\dot{z}_{2}(t)=-\mu z_{2}(t)+\mu z_{2}(t-\tau),
\end{array}
$$

where $z_{1} \in R^{N-1}, z_{2} \in R$ and the matrix $B$ in given in (10).

\section{Proof: Consider system (9)}

$\left[\begin{array}{c}\dot{z}_{1}(t) \\ \dot{z}_{2}(t)\end{array}\right]=\left[\begin{array}{cc}B & \overrightarrow{0} \\ \overrightarrow{0}^{T} & 0\end{array}\right]\left[\begin{array}{l}z_{1}(t) \\ z_{2}(t)\end{array}\right]-\left[\begin{array}{c}A_{1}^{\prime} \\ A_{2}^{\prime}\end{array}\right] \int_{t-\tau}^{t} \dot{z}(s) d s$,

where $\left[\begin{array}{l}A_{1}^{\prime} \\ A_{2}^{\prime}\end{array}\right]=U A W$ and $A_{2}^{\prime}=(U A W)_{N}$. The system can be split into two equations where the vector $z_{1}$ contains the $N-1$ first components of $z$ and $z_{2}$ is equal to the last component of $z$. Then we have:

$$
\begin{gathered}
\dot{z}_{1}(t)=B z_{1}(t)-A_{1}^{\prime} \int_{t-\tau}^{t} \dot{z}(s) d s, \\
\dot{z}_{2}(t)=A_{2}^{\prime} \int_{t-\tau}^{t} \dot{z}(s) d s .
\end{gathered}
$$

The stability of this time delay system has to be examined with respect to the values of the delay $\tau$. However this is not an easy task since the integral terms depends on $z$ and not on $z_{1}$ and $z_{2}$ in the first and the second equation respectively. From (10), simple manipulations leads to:

$$
\left[\begin{array}{l}
A_{1}^{\prime} \\
A_{2}^{\prime}
\end{array}\right]=U A W=\left[\begin{array}{cc}
B+\mu I & \overrightarrow{0} \\
\overrightarrow{0}^{T} & \mu
\end{array}\right] .
$$

System (12) can now be written as:

$$
\begin{aligned}
& \dot{z}_{1}(t)=B z_{1}(t)-(B+\mu I) \int_{t-\tau}^{t} \dot{z}_{1}(s) d s, \\
& \dot{z}_{2}(t)=-\mu \int_{t-\tau}^{t} \dot{z}_{2}(s) d s,
\end{aligned}
$$

or in the time delay representation (11).

The consensus problem has now been rewritten in an appropriate form to develop stability criteria.

Remark 5: Note that the variable $z_{2}$ is defined by

$$
z_{2}(t)=U_{2} x(t)
$$

In the case of a symmetric network, the matrix $W$ is an orthogonal matrix which means $U=W^{T}$. Then if the last column of $W$ is $\alpha \overrightarrow{\mathbf{1}}$, then $U_{2}=1 /(\alpha N) \overrightarrow{\mathbf{1}}$, which means that $z_{2}$ corresponds to the average of the position of all agents. This does not hold for an asymmetric communication network.

\section{STABILITY ANALYSIS}

This section focuses on the stability of the consensus problem (11). We first check the convergence of the second subsystem of (11b). An expression of the consensus equilibrium will be given. Then two approaches using a frequency approach, for the symmetric case, and a time domain approach for the symmetric and non symmetric cases will be provided.

\section{A. Consensus equilibrium}

Lemma 3: The system (11b) is stable for any delay $\tau$ and converges to

$$
z_{2 e q}=\lim _{s \rightarrow 0} s \frac{z_{2}(0)+\mu e^{-\tau s} \int_{-\tau}^{0} z_{2}(u) e^{-u s} d u}{s+\mu\left(1-e^{-\tau s}\right)} .
$$

Proof: Consider the second sub-system (11b). It can easily and more efficiently analyzed using a frequency approach. The Laplace transform of system (11b) is:

$$
\begin{aligned}
s Z_{2}(s)-z_{2}(0)= & -\mu Z_{2}(s)+\mu e^{-\tau s} Z_{2}(s) \\
& +\mu \int_{-\tau}^{0} z_{2}(u) e^{-(u+\tau) s} d s .
\end{aligned}
$$

Thus we have:

$$
Z_{2}(s)=\frac{z_{2}(0)+\mu \int_{-\tau}^{0} z_{2}(u) e^{-(u+\tau) s} d s}{s+\mu\left(1-e^{-\tau s}\right)} .
$$

The stability of (11b) is determined by the roots of the equation:

$$
s+\mu\left(1-e^{-\tau s}\right)=0 .
$$

Consider $s=\alpha+j \beta$ with $\alpha, \beta \in R$. Then the last equation yields:

$$
\begin{aligned}
\alpha+\mu-\mu e^{-\alpha \tau} \cos (\beta \tau) & =0, \\
\beta+\mu e^{-\alpha \tau} \sin (\beta \tau) & =0,
\end{aligned}
$$

Note that $s=0$ is a solution of (14). However if $\beta$ satisfies (14b), $-\beta$ also does. For all $\beta$ such that $\beta \tau=k \pi$ with $k \in N /\{0\},(14 \mathrm{~b})$ does not hold. This means that $\cos (\beta \tau) \neq$ $1,-1$. For all the solutions $\beta_{k}, k \in N /\{0\}$, of (14b), define $\left.\epsilon_{k} \in\right]-1,1\left[\right.$ such that $\epsilon_{k}=\cos \left(\beta_{k} \tau\right)$. If $\epsilon_{k} \leq 0$, then 
$\alpha \leq-\mu$. If $\epsilon_{k}>0$, consider the function $f_{\epsilon_{k}}(\alpha)=\alpha+\mu-$ $\epsilon_{k} \mu e^{-\alpha \tau}$. As $\epsilon_{k}>0, f_{\epsilon_{k}}$ is a strictly increasing function. By noting that $f_{\epsilon_{k}}(0)=\mu\left(1-\epsilon_{k}\right)>0$, this means that the solutions $\alpha_{k}$ of (14a) such that $f_{\epsilon_{k}}\left(\alpha_{k}\right)=0$ are strictly negative.

Then all the roots of (14) are such that $\alpha \leq 0$. From [15], the system $(11 \mathrm{~b})$ is stable and the final equilibrium of $z_{2}$ is given by:

$$
\lim _{t \rightarrow \infty} z_{2}(t)=\lim _{s \rightarrow 0} s \frac{z_{2}(0)+\mu \int_{-\tau}^{0} z_{2}(u) e^{-(u+\tau) s} d u}{s+\mu\left(1-e^{-\tau s}\right)} .
$$

\section{B. Main result}

Theorem 1: Consider the system (7) with a constant delay $\tau$. If there exists $\bar{\tau} \geq \tau$ such that:

- $1+\lambda_{i}(-B-\mu I) \frac{1-e^{s \bar{\tau}}}{s} \neq 0$, for all $s \in C^{+}$, and

- there exist symmetric and positive-definite matrices: $P, S$ such that the following LMI holds:

$$
\left[\begin{array}{cc}
B^{T} P+P B+\bar{\tau} S & B^{T} P(B+\mu I) \\
(B+\mu I)^{T} P B & -\bar{\tau} S
\end{array}\right]<0,
$$

then all elements of $x$ converge asymptotically to a common valus $x_{e q}$ which is given by:

$$
x_{e q}=U_{2}\left(\lim _{s \rightarrow 0} s \frac{x(0)+\mu e^{-\tau s} \int_{-\tau}^{0} x(u) e^{-u s} d u}{s+\mu\left(1-e^{-\tau s}\right)}\right) \overrightarrow{\mathbf{1}} .
$$

Proof: Consider the consensus problem (8) under a symmetric or non-symmetric communication network and a constant delay $\tau$. There exists a change of coordinates $z=$ $W x$, where $W$ is an orthogonal matrix in the symmetric case or a non singular matrix in the non symmetric case, such that the system can be rewritten as (11). The first part of the proof is to show that the reduced-order variable $z_{1}$ is stable. Consider thus the reduced-order system:

$$
\dot{z}_{1}(t)=-\mu I z_{1}(t)+(B+\mu I) z_{1}(t-\tau) .
$$

If Lemma 1 is satisfied for system (16) with $A_{0}=-\mu I$, $A_{1}=B+\mu I$ and $\tau$, then $z_{1}$ converges to $z_{1}=0$. Finally according to Lemma 3 and the change of coordinates defined by $W$ and $U$, the equilibrium is given in Theorem 1 .

Note that the stability conditions does not depend on the choice of $W$. It is only required that $B$ belongs to $R^{(N-1) \times(N-1)}$.

In [10], it was noted that the consensus problem (6) does not preserve the average consensus. In contrast to [7], [8], the effect that initial conditions and delays have in the resulting consensus equilibrium is explicitly shown.

Another issue that has to be solved concerns the case of disconnected communication networks. In the case of a disconnected network, $B$ has at least one 0-eigenvalue [3]. Then the stability conditions given in Lemma 1 will not be satisfied as well since the matrix $B$ is not Hurwitz. The term $B^{T} P+P B$ can not be negative definite and consequently the LMI (15) can not hold. It also means that there exists at least another eigenvalue equal to zero. Then another equation like (11b) will define another equilibrium.

From Theorem 1, it can be seen that the initial condition has a strong effect on the equilibrium position. The following corollaries examine two different cases of initial conditions:

Corollary 1: Consider initial conditions of the form:

$$
\begin{aligned}
& x(\theta)=0, \quad \forall \theta \in[-\tau, 0[ \\
& x(0)=x_{0} \neq 0,
\end{aligned}
$$

Then the equilibrium is given by :

$$
x_{e q}=U_{2} x_{0} /(1+\mu \tau) \overrightarrow{1} \text {. }
$$

It can then be seen that the delay is attenuated in the value of the final equilibrium. Note that in this context, we are not considering discontinuous initial conditions but discontinuity in the control law (2).

Corollary 2: Consider initial conditions of the form:

$$
x(\theta)=x_{0}, \quad \forall \theta \in[-\tau, 0],
$$

Then the equilibrium is

$$
x_{e q}=U_{2} x_{0} \overrightarrow{1}
$$

Proof: The result is straightforward by noting that:

$$
\int_{-\tau}^{0} z_{2}(u) e^{-(u+\tau) s} d u=\mu z_{2}(0) / s\left(1-e^{-\tau s}\right) .
$$

The initial condition did indeed not change the final equilibrium of the consensus problem. However in this case, the position of the equilibrium did not change compared to the non delay case.

Note that these two examples are motivated by practical considerations. Corollary 1 implies that, whatever the position of all the agents, the control laws $u_{i}(t)$ will only use information taken after the initial time $t=0$. During $[0, \tau]$, no information from the other agents is used in the control. On the other hand, Corollary 2 can be interpreted as follows. At time $t=-\tau$, all agents have to wait until they receive data from their neighbors. At time $t=0$, the control using non zero initial conditions in $[-\tau, 0]$ is implemented.

Finally, the difference between these two protocols has an unexpected influence on the position of the equilibrium point.

\section{Precision on the symmetric case}

Provided that the communication graph is connected, the rest of the Laplacian eigenvalues are all positive. We denote them by $0<\lambda_{2} \leq \ldots \leq \lambda_{N}$. It thus means that $B$ is a diagonal matrix with $-\lambda_{i}$. The first equation of (11) can be further decomposed into $N-1$ equations based on the diagonal form of the $B$ matrix. In particular, denoting $z_{1}=$ $\left[z_{11}, \ldots, z_{1, N-1}\right]$ the first equation of (11) is equivalent to

$\dot{z}_{1 i}(t)=-\mu z_{1 i}(t)+\left(-\lambda_{i}+\mu\right) z_{1 i}(t-\tau), i \in\{2, \ldots, N\}$.

In the sequel, we examine the stability of the system (17). 
Theorem 2: The consensus problem (9) is asymptotically stable for all delays $\tau$ and the consensus equilibrium is given by:

$$
x_{e q}=\lim _{s \rightarrow 0} s \sum_{i=1}^{N}\left(\frac{x_{i}(0)+\mu e^{-\tau s} \int_{-\tau}^{0} x_{i}(u) e^{-u s} d u}{s+\mu\left(1-e^{-\tau s}\right)}\right) \overrightarrow{\mathbf{1}} .
$$

Proof: The proof follows the line of the proof of Theorem 1. Consider equation (17) in the frequency domain. Its stability is equivalent to proving that all the roots of the characteristic equation:

$$
s+\mu+\left(\lambda_{i}-\mu\right) e^{-\tau s}=0,
$$

lie in the left hand side of the complex plane. Consider $s=$ $\alpha+j \beta$ with $\alpha, \beta \in R$. Then (19) is equivalent to:

$$
\begin{aligned}
\alpha+\mu+\left(\lambda_{i}-\mu\right) e^{-\alpha \tau} \cos (\beta \tau) & =0, \\
\beta-\left(\lambda_{i}-\mu\right) e^{-\alpha \tau} \sin (\beta \tau) & =0,
\end{aligned}
$$

It is well known that these equations have an infinite number of solutions, and that the solutions are conjugate, i.e., if $\beta$ is a solution of (20b) then $-\beta$ is a solution as well.

For all $\beta_{k}$ which are solutions of (20b), define $\epsilon_{k}=$ $\cos \left(\beta_{k} \tau\right)$. Since (20b) is verified, $\epsilon_{k}$ can not be equal to 1 or -1 , except the case $\beta=0$. If $\epsilon_{i}\left(\lambda_{i}-\mu\right) \leq 0$, then $\alpha+\mu \leq 0$. Then $\alpha$ is strictly negative. For $\epsilon_{k}\left(\lambda_{i}-\mu\right)>0$, consider the function $f_{\epsilon_{k}}=\alpha+\mu-\epsilon_{k}\left(\lambda_{i}-\mu\right) e^{-\alpha \tau}$. Since $\epsilon_{k}\left(\lambda_{i}-\mu\right)>0, f_{\epsilon_{k}}$ is a strictly increasing function. Consider now $f_{\epsilon_{k}}(0)=\mu+\epsilon_{k}\left(\lambda_{i}-\mu\right)$. From [5], the eigenvalues of $B$ lie in the interval $] 0,2 \mu]$. This implies that $\left|\lambda_{i}-\mu\right| \leq \mu$. Since $\epsilon_{k}$ lies in ] $-1,1\left[, f_{\epsilon_{i}}\right.$ is strictly positive. It means that the solution $\alpha_{k}$ such that $f_{\epsilon_{k}}\left(\alpha_{k}\right)=0$ is strictly negative. Since all the roots of (19) have strictly negative real part, the solutions asymptotically converge to $z_{i 1}=0$, whatever the delay $\tau$. Finally according to Lemma 3, the equilibrium is given by (13) and is further simplified using $U_{2}=1 /(\alpha N) \overrightarrow{1}$.

\section{EXAMPLE}

Consider a set four agents moving from their initial positions $X_{0}=\left[\begin{array}{llll}0 & 5 & 15 & 20\end{array}\right]$. We consider different kind of networks and wether or not consensus is achieved and then provide the consensus value. The communication networks are defined with $\mu=1$ and the following adjacency matrices:

$$
\begin{gathered}
A_{0}=\left[\begin{array}{cccc}
0 & 0.5 & 0 & 0.5 \\
0.5 & 0 & 0.5 & 0 \\
0 & 0.5 & 0 & 0.5 \\
0.5 & 0 & 0.5 & 0
\end{array}\right], A_{1}=\left[\begin{array}{cccc}
0 & 1 & 0 & 0 \\
0.5 & 0 & 0 & 0.5 \\
0 & 0.5 & 0 & 0.5 \\
0 & 0.5 & 0.5 & 0
\end{array}\right], \\
A_{2}=\left[\begin{array}{cccc}
0 & 1 & 0 & 0 \\
0 & 0 & 1 & 0 \\
0 & 0 & 0 & 1 \\
1 & 0 & 0 & 0
\end{array}\right], A_{3}=\left[\begin{array}{cccc}
0 & 1 & 0 & 0 \\
1 & 0 & 0 & 0 \\
0 & 0 & 0 & 1 \\
0 & 0 & 1 & 0
\end{array}\right], \\
A_{4}=\left[\begin{array}{cccc}
0 & 1 / 3 & 1 / 3 \\
1 / 3 & 0 & 1 / 3 & 1 / 3 \\
1 / 3 & 1 / 3 & 0 & 1 / 3 \\
1 / 3 & 1 / 3 & 1 / 3 & 0
\end{array}\right] .
\end{gathered}
$$

Note that $A_{0}$ and $A_{4}$ are a symmetric matrices, $A_{1}$ and $A_{2}$ are non-symmetric and $A_{3}$ represents a disconnected graph. The following table shows the coordinates of the consensus equilibrium provided that the LMI of Theorem 2 are satisfied.
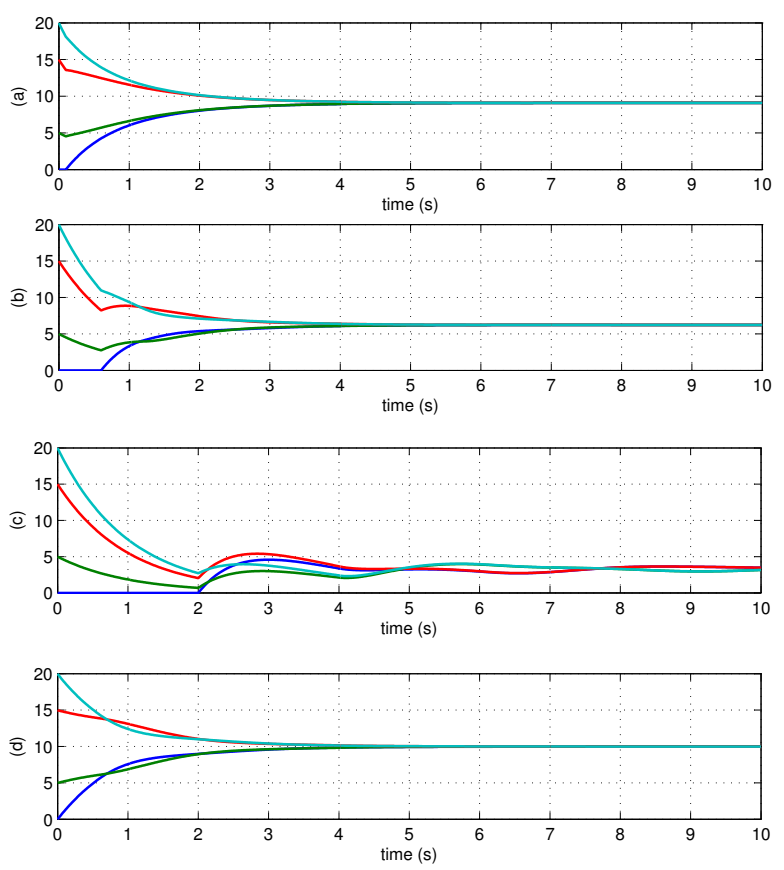

Fig. 1. Simulation results for $A_{0}$ and Corollary 1 type with $\tau=0.1$ (a), $\tau=0.6$ (b) and $\tau=2$ (c) and Corollary 2 with $\tau=0.6$ (d)

The conditions of Theorem 1 can be verified for all delay bounds $\bar{\tau}$, which means that consensus is achieved for any delay $\tau$.

\begin{tabular}{|l|c|c|c|c|c|}
\hline Network & $A_{0}$ & $A_{1}$ & $A_{2}$ & $A_{3}$ & $A_{4}$ \\
\hline $\bar{\tau}$ & $\infty$ & $\infty$ & $\infty$ & $\mathrm{X}$ & $\infty$ \\
\hline $\begin{array}{l}C 1 \& \\
\tau=0.1\end{array}$ & 9.09 & 8.48 & 9.09 & $\mathrm{X}$ & 9.09 \\
\hline $\begin{array}{l}C 1 \& \\
\tau=0.6\end{array}$ & 6.25 & 5.83 & 6.25 & $\mathrm{X}$ & 6.25 \\
\hline $\begin{array}{l}C 1 \& \\
\tau=2\end{array}$ & 3.33 & 3.11 & 3.33 & $\mathrm{X}$ & 3.33 \\
\hline$C 2$ & 10 & 9.33 & 10 & $\mathrm{X}$ & 10 \\
\hline
\end{tabular}

Figures 1, 2 and 3 show the simulation results for the consensus defined by the adjacency matrices $A_{0}, A_{1}$ and $A_{4}$. It contains three different cases which correspond to the initial conditions of Corollary 1 type with $\tau=0.1$ (a), $\tau=0.6$ (b) and $\tau=2$ (c) and Corollary 2 type with $\tau=0.6$ (d). For all of them, consensus is achieved, but the consensus equilibria are different.

In all the figures, the plots (a), (b) and (c) show the influence the initial conditions on the solutions. It can be seen that the agents are only driven by the diagonal terms during the interval $[0, \tau]$. Then since the delayed terms act on the dynamics, the agents achieve a consensus.

In the (c) plots, consensus is achieved with the classical oscillatory behavior of time-delay systems.

Another interesting comment concerns the convergence rate. Consider the cases where the initial conditions follow Corollary 1 in Figure 3. It can be seen that the convergence 

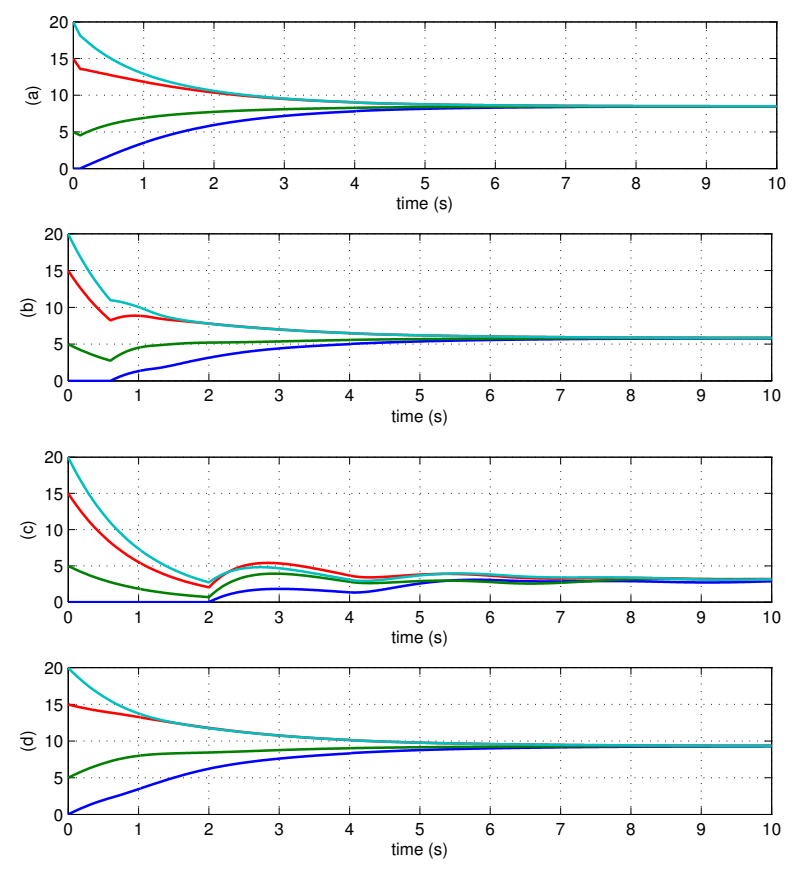

Fig. 2. Simulation results for $A_{1}$ and the same cases as in Figure 1.

rate for $\tau=0.1$ and $\tau=1.2$ is less than the one for $\tau=0.6$. Thus, it is not intuitive that the introduction of a delay into the consensus problem may improve the convergence rate. The phenomenon does not appear in the simulations with $A_{1}$ and $A_{0}$ which means that the behavior depends on the network. Some recent articles already investigated this issue [6],[14]. Further research investigating on the influence of the delay on the convergence rate would be interesting to explain these phenomena.

\section{CONClusion}

The influence of time delays in the consensus problem was studied. The main result shows that consensus is achieved but the position of equilibrium point strongly depends both on the value of the delay and on the initial conditions. This time delay approach allows considering simple symmetric/asymmetric and connected/disconneted communication network. Further research involves considering different time-varying delays and using results on exponential stability of time-delay systems [13] to provide an estimate of the exponential decay rate.

\section{REFERENCES}

[1] D.V. Dimarogonas and K.J. Kyriakopoulos, A connection between formation infeasibility and velocity alignment in kinematic multi-agent systems, Automatica, to appear, 2008.

[2] E. Fridman, A. Seuret and J.-P. Richard, Robust sampled-data stabilization of linear systems: An input delay approach, Automatica, Vol. 40(8), p.1441-1446, 2004.

[3] C. Godsil and G. Royle, Algebraic Graph Theory, Springer Graduate Texts in Mathematics \# 207,2001.

[4] K. Gu, V.L. Kharitonov and J. Chen, Stability of time-delay systems, Birkhauser, 2003

[5] R.A. Horn and C.R. Johnson, Matrix analysis, Cambridge, UK Cambridge Univ. Press, 1987.
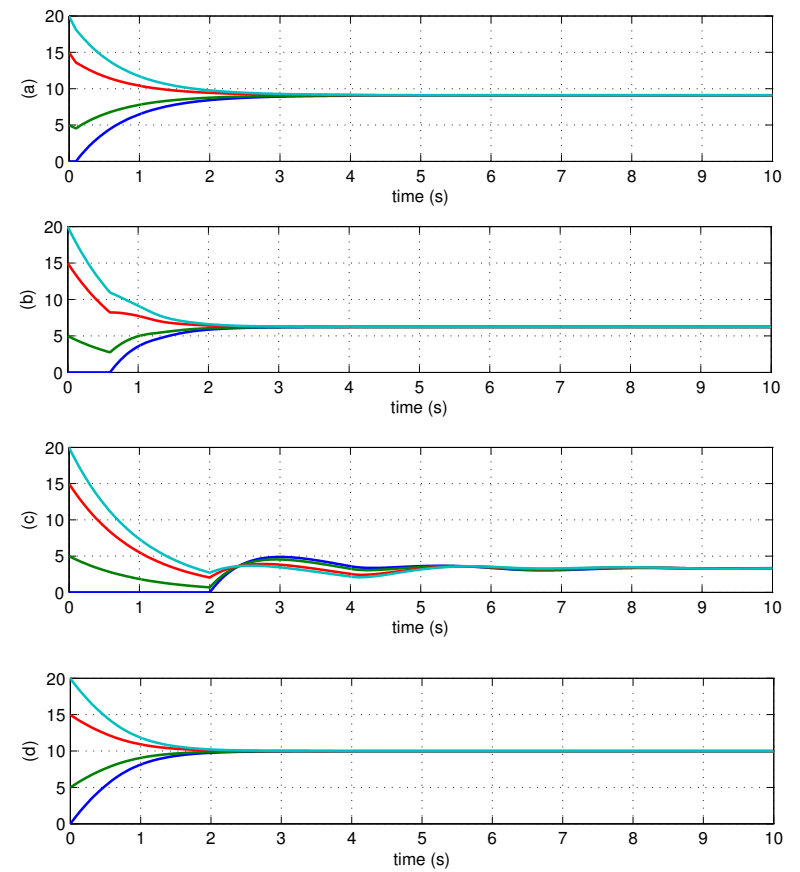

Fig. 3. Simulation results for $A_{4}$ and different values of the delay.

[6] W. Michiels and S.-I. Niculescu and L. Moreau, Using delays and time-varying gains to improve the statc output feedback stabilizability of linear systems : a comparison, IMA Journal of Mathematical Control and Information, Vol. 21(4), pp.393-418, 2004.

[7] L. Moreau, Stability of continuous-time distributed consensus algorithms, 43rd IEEE Conference on Decision and Control, 2004.

[8] L. Moreau, Stability of multi-agent systems with time-dependent communication links, IEEE Trans. on Automatic Control, vol. 50, pp. 169-182, February 2005.

[9] S.-I. Niculescu, Delay Effects on Stability. A Robust Control Approach, Springer-Verlag, 2001.

[10] R. Olfati-Saber and A. Fax and R.M. Murray, Consensus and cooperation in networked multi-agent systems, Proceedings of the IEEE, vol. 95(1), January 2007.

[11] R. Olfati-Saber and R.M. Murray, Consensus Problems in network of agents with switching topology and time delays, IEEE Transactions on Automatic Control, vol. 49(9), September 2004.

[12] J.-P. Richard, Time delay systems: an overview of some recent advances and open problems, Automatica, vol. 39(10), 2003, pp 16671694.

[13] A. Seuret and M. Dambrine and J.P. Richard, Robust exponential stabilization for systems with time-varying delays, $5^{\text {th }}$ Workshop on Time Delay Systems, 2004, Leuven, Belgique.

[14] A. Seuret, C. Edwards, S.K. Spurgeon and E. Fridman, Static output feedback sliding mode control design via an artificial stabilizing delay, accepted for publication to IEEE Trans on Aut. Control, 2007.

[15] G. Stépán, Retarded dynamical systems: stability and characteristic function, Research Notes in Math. Series,210, Longman Scientific, UK, 1989.

[16] H.G. Tanner and D.K. Christodoulakis, The stability of synchronization in local-interaction networks is robust with respect to time delays, 44th IEEE Conference on Decision and Control, p.49454950, 2005.

[17] L. Xiao and S. Boyd, Fast Linear Iterations for Distributed Averaging, it 42nd IEEE Conf. Decision and Control, p. 4997-5002, 2003. 